\title{
Apolipoprotein E4 Genotype Increases the Risk of Being Diagnosed With Posttraumatic Fibromyalgia
}

\author{
Jonathan C. Reeser, MD, PhD, Erin Payne, MPH, Terrie Kitchner, \\ Catherine A. McCarty, PhD, MPH
}

Objective: To determine whether the apolipoprotein E4 (Apo E4) allele may be a genetic risk factor for fibromyalgia syndrome (FMS).

Design: A retrospective assessment of associations between Apo E4 genotype and selected environmental exposures among a cohort diagnosed with FMS compared with control subjects. Setting: Marshfield Clinic Research Foundation's Personalized Medicine Research Project (PMRP) biobank.

Participants: One hundred fifty-one case subjects with fibromyalgia and 300 age- and gender-matched control subjects.

Methods: Fibromyalgia case subjects were identified according to a strict phenotypic definition from among the nearly 20,000 subjects enrolled in the PMRP. Age- and gendermatched control subjects also were identified from the PMRP in a 2:1 control/case ratio. Apo E4 genotype was determined by single nucleotide polymorphism analysis for both case subjects with fibromyalgia and control subjects. Case subjects with fibromyalgia and control subjects were asked to assess their level of function and stress by completing the Short Form-36 and the Perceived Stress Scale.

Main Outcome Measures: Statistical associations between the Apo E4 genotype and phenotypic criteria (diagnosis of FMS) as well as historical environmental exposures as documented in the electronic medical record were assessed.

Results: Approximately one quarter of both case subjects with fibromyalgia and control subjects were found to carry at least one Apo E4 allele. The odds ratio (OR) for case subjects with fibromyalgia who had ever been in a motor vehicle accident and subsequently had been diagnosed with FMS was increased among those with at least one copy of the Apo E4 allele (OR 7.04) compared with those without an Apo E4 allele (OR 1.90). The presence of an Apo E4 allele did not influence the degree of pain or level of function among those with FMS. Conclusions: These data suggest that specific interactions between genetically susceptible individuals (eg, those with at least one copy of the Apo E4 allele) and the environment (eg, involvement in a motor vehicle accident) may contribute to the risk of being diagnosed with FMS, although Apo E4 allele status does not appear to modulate perceived FMS severity.

PM R 2011;3:193-197

\section{INTRODUCTION}

The fibromyalgia syndrome (FMS) is a chronic pain condition that has been estimated to affect $2 \%$ to $6 \%$ of the U.S. population. More than $80 \%$ of those diagnosed with FMS are women. Characteristic symptoms include diffuse (typically symmetric) soft-tissue pain, persistent fatigue, headaches, cognitive or memory impairment, morning stiffness, and nonrestorative sleep, in addition to irritable bowel syndrome, irritable bladder, depression, and temporomandibular joint discomfort. The diagnosis of FMS is determined solely by historical information and physical examination findings. At present no "objective" diagnostic laboratory tests exist to diagnose FMS; likewise, no universally effective treatments exist [1].

Despite its apparent prevalence, a residual undercurrent of skepticism exists in the broader medical community regarding the validity of the fibromyalgia construct. This skepticism may reflect our relatively rudimentary understanding of the pathophysiology underlying FMS. In that regard, debate continues over whether FMS is a valid and unique clinical entity. FMS shares a remarkable number of features with overtraining syndrome, chronic fatigue syndrome, and
J.C.R. Office of Research Integrity and Protections, Marshfield Clinic Research Foundation, 1000 North Oak Ave, Marshfield, WI 54449 Address correspondence to J.C.R.; e-mail: reeser.jonathan@marshfieldclinic.org Disclosure: nothing to disclose

E.P. Department of Epidemiology, School of Public Health, University of Michigan, Ann Arbor, Ml

Disclosure: nothing to disclose

T.K. Department of Human Genetics, Marshfield Clinic Research Foundation, Marshfield, WI Disclosure: nothing to disclose

C.A.M. Department of Human Genetics, Marshfield Clinic Research Foundation, Marshfield, WI

Disclosure: nothing to disclose

Peer reviewers and all others who control content have no relevant financial relationships to disclose.

Disclosure Key can be found on the Table of Contents and at www.pmrjournal.org This investigation was funded by a grant from the Marshfield Clinic Research Foundation's Physician Research Fund.

Submitted for publication August 27, 2010, accepted December 3, 2010. 
Table 1. Comparison of clinical features of FMS and PCS

\begin{tabular}{lll}
\hline & FMS & PCS \\
\hline Fatigue & +++ & +++ \\
Headache & ++ & +++ \\
IBS & + & +++ \\
Impaired cognition & + & ++ \\
Mood disturbance & ++ & + \\
Morning stiffness & + & + \\
Myalgias & +++ & ++ \\
Sleep dysfunction & +++ & \\
TMJ & + & \\
\hline
\end{tabular}

Data compiled from Kato et al [2] and Johnston et al [3].

$+++=$ Nearly universal complaint for this diagnosis.

$++=$ Very common complaint for this diagnosis.

$+=$ Occasional complaint for this diagnosis.

$\mathrm{FMS}=$ fibromyalgia syndrome; IBS = irritable bowel syndrome; PCS = postconcussive syndrome; TMJ = temporomandibular joint discomfort.

(perhaps most strikingly) postconcussive syndrome (PCS; Table 1) [2]. Although the shared features of these diagnoses are readily apparent, no clear-cut mechanistic basis for the similarity between these clinical conditions is immediately obvious.

PCS comprises a constellation of symptoms that occur on a relatively common basis after mild traumatic brain injury $[3,4]$. Like FMS, PCS is characterized by mood disturbance, myalgias, disordered sleep, cognitive impairment, and headaches. Risk factors for PCS include female gender, age, and a history of inciting trauma. Research has revealed that the apolipoprotein E4 (Apo E4) genotype is associated with a poorer outcome from PCS [5]. In addition, Apo E4 may be thought of as a risk factor for the development of PCS because persons positive for Apo E4 have a greater incidence of PCS after mild traumatic brain injury $[6,7]$.

Evidence suggests that FMS is a neurophysiologic condition involving a disordered perception of pain [8]. Environmental triggers such as stress and trauma may play a critical role in the development of fibromyalgia, in addition to potentially modulating the severity of symptoms experienced. The tendency for the syndrome to cluster in families suggests that a genetic factor contributes to the risk of developing fibromyalgia $[9,10]$. Arnold et al [1] demonstrated that first-degree relatives of individuals diagnosed with FMS were more likely to be diagnosed with FMS. However, it is evident that FMS does not follow a simple Mendelian inheritance pattern; multiple genes probably contribute to its etiopathogenesis. Although a unique genotype has not been definitively associated with FMS, genes that have been implicated in the diagnosis include those involved in serotonin neurotransmission and neuropeptide synthesis [11].

Apo $E$ is a polymorphic molecule found as a component of many lipoproteins. In the central nervous system, Apo E is produced by astrocytes and microglia and has been proposed to play a role in transporting lipids from astrocytes to injured neurons for repair. Apo E also is involved in synaptogenesis, clearance of degeneration products, microglial activation, and maintenance of the cholinergic system [12]. Three known human Apo E isoforms exist-E2, E3, and E4 - that correspond to their respective genes-Apo E $\varepsilon 2$, Apo E $\varepsilon$ 3, and Apo E $\varepsilon 4$. In light of the striking clinical similarities between FMS and PCS, we concluded that it would be reasonable to investigate whether an association also exists between Apo E4 genotype and FMS. This short report documents our findings, which suggest that the Apo E4 allele, when combined with a history of having been involved in a motor vehicle accident (MVA), is associated with an increased risk of being diagnosed with posttraumatic FMS.

\section{METHODS}

Both the Research Committee and the Institutional Review Board of the Marshfield Clinic approved the project. A total of 151 case subjects and 300 age- and gender-matched control subjects were identified from within the Marshfield Clinic Research Foundation's Personalized Medicine Research Project (PMRP) database. The PMRP is one of the United State's largest biobanks, with an enrollment of approximately 20,000 [13]. All of the participants in the PMRP reside within the catchment area of the Marshfield Clinic (Figure 1), and the vast majority receive their health care from Marshfield Clinic (which has a searchable electronic medical record [EMR]).

Potential subjects were identified by screening the EMR for individuals who had received the diagnosis of FMS at least twice during a 6-month period, then cross-referencing those identified cases with enrollment in the PMRP. Potential control subjects were similarly identified, excluding individuals who had ever received the diagnosis of FMS. Individual charts were subsequently manually abstracted to confirm the participant's phenotype (ie, either case or control) using the following criteria:

- Case subjects were $\geq 18$ years of age with documented chronic widespread pain and tenderness. Most commonly the diagnosing physician was a rheumatologist, physiatrist, or family practitioner. Although the style and thoroughness of symptom documentation varied, in every instance multiple areas of pain were described, typically involving both sides of the body. In addition, the EMR was reviewed to identify any associated symptoms consistent with the diagnosis of FMS

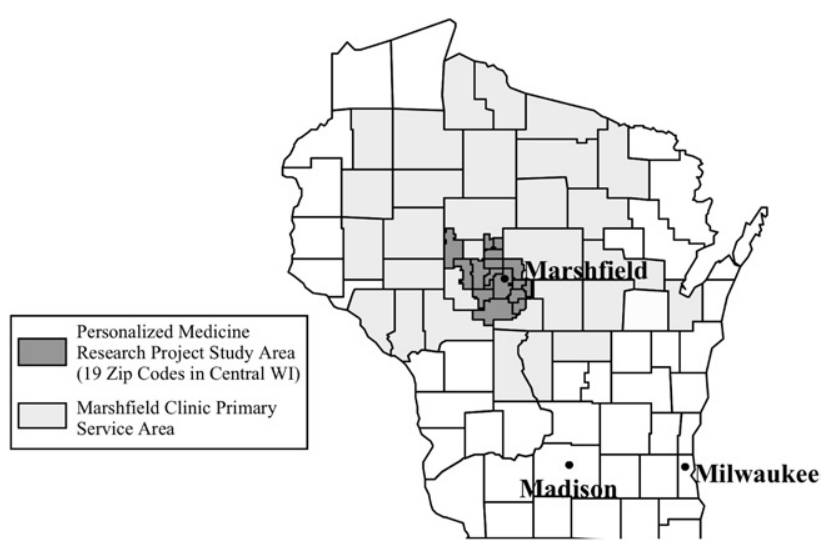

Figure 1. The Personalized Medicine Research Project catchment area. 
and to confirm that the potential subject had no history of concomitant rheumatologic condition that might otherwise explain their symptoms.

- Control subjects were $\geq 18$ years of age with no diagnosis of FMS or other rheumatologic disease (including discrete myofascial pain).

The methods for separating cells and purifying and storing deoxyribonucleic acid obtained from PMRP participants are described elsewhere [14]. Genotyping analysis of the case and control subjects was performed with use of single nucleotide polymorphism (SNP) primer extension/polymerase chain reaction methodology. The SNPs used to determine the Apo E genotype included rs7412 and rs429358.

Information regarding each subject's documented history of motor vehicular trauma, tobacco use, and body mass index was extracted from the EMR. In addition, all subjects with FMS and the associated control subjects were recontacted and invited to provide additional information regarding their current average monthly pain (assessed by a 100-mm visual analogue scale [VAS]) and perceived stress levels (assessed by the 10-item Perceived Stress Scale [PSS]). Higher scores on the PSS indicate greater levels of perceived stress [15]. The Short Form-36 (SF36) was administered as a means of assessing overall quality of life/perceived health status [16]. Eight composite scores and 2 summary measures (each scored from 0 to 100 , with higher scores indicating a higher quality of life/health status) were derived from the data collected from each respondent.

\section{Statistics}

Statistical analysis was performed according to the purposes of the study with use of SPSS version 15.0 statistical software (SPSS, Chicago, IL). $\chi^{2}$ Analyses were performed to compare the categorical variables, whereas $t$-tests were used to compare continuous variables. A $P$ value $<.05$ was interpreted as an indication of statistical significance. Multivariate analysis was performed to examine the association between the selected potential environmental and genetic risk factors and the FMS phenotype.

\section{RESULTS}

\section{The PMRP FMS Cohort}

We identified an experimental cohort from the PMRP biobank consisting of 151 FMS case subjects and 300 age- and gendermatched control subjects. The FMS case subjects ranged in age from 20 to 80 years, with a mean of 53.1 years. Control subjects were age-matched within 5 years, with a range from 20 years to 81 years, and a mean of 52.7 years. Men comprised only $4 \%$ of the case and control subjects within the cohort. We observed no significant difference between the mean body mass index for case and control subjects ( 31 and 29, respectively). The prevalence of various symptoms within the case group was calculated to be as follows: diffuse myofascial pain, $100 \%$; sleep disturbance, 97\%; fatigue, 90\%; headache, $87 \%$; depression, $79 \%$; paresthesias,
Table 2. Multivariate associations between selected environmental interactions, number of Apo E4 alleles, and the risk of being diagnosed with FMS

\begin{tabular}{lcc}
\hline & $\begin{array}{c}\text { No Apo E4 Alleles } \\
\text { Odds Ratio } \\
\text { (95\% Cl) }\end{array}$ & $\begin{array}{c}\mathbf{1 - 2} \text { Apo E4 Alleles } \\
\text { Odds Ratio } \\
\text { (95\% Cl) }\end{array}$ \\
\hline Ever smoked & $2.25(1.33,3.83)$ & $2.29(0.82,6.33)$ \\
Ever experienced & $1.90(1.03,3.54)$ & $7.04(1.98,25.0)$ \\
an MVA & & \\
\hline
\end{tabular}

Apo $=$ apolipoprotein; $\mathrm{Cl}=$ confidence interval; $\mathrm{FMS}=$ fibromyalgia syndrome; MVA = motor vehicle accident.

65\%; temporomandibular joint discomfort, $24 \%$; irritable bowel or bladder, 20\%; and cognitive impairment, $18 \%$.

\section{Apo E4 Genotype}

The percentage of the cohort found to carry at least one Apo E4 allele was $26.8 \%$, although no significant difference existed between the prevalence of the Apo E4 allele among case subjects (26.0\% had at least one allele) and control subjects (27.3\% had at least one allele). The percentage of FMS case subjects who reported "ever smoking" was $41 \%$ compared with $24 \%$ of control subjects $(P<.001)$, but when stratified by Apo E4 status, a history of ever smoking did not significantly affect the odds ratio (OR) (OR 2.25 with no Apo E4 allele versus 2.29 for those with 1-2 copies; Table 2). We also evaluated the cohort for a documented history of MVA and found that 34\% of those diagnosed with FMS had ever been in an MVA compared with 14\% of the control group $(P<.001)$. Among those with a history of MVA, the risk of being diagnosed with FMS was substantially greater among the subjects who carried at least one Apo E4 allele (OR 7.04) compared with those without an Apo E4 allele (OR 1.90; Table 2). Unfortunately, the number of case subjects carrying one- 2 Apo E4 alleles who had ever smoked and who had a history of MVA was too small to permit computation of a meaningful OR.

\section{Stress and Pain}

We explored the role of stress and Apo E4 genotype status in the clinical manifestation of FMS by administering the SF-36 and the 10-item PSS to the PMRP cohort. One hundred thirty-three case subjects and 247 control subjects (88\% and $82 \%$ response rate, respectively) returned completed instruments. Respondents also estimated their average monthly pain with use of a 100-mm VAS. As summarized in Table 3, those diagnosed with FMS perceived greater stress, reported greater average monthly pain, and had lower SF-36 Physical and Mental Component Summary scores compared with control subjects. As expected, the average pain score and the level of perceived stress were significantly greater among those with FMS compared with control subjects. However, Apo E4 status was not statistically associated with any of the outcome measures used to quantify stress or pain. 
Table 3. Measures of stress and pain among the PMRP FMS cohort

\begin{tabular}{|c|c|c|c|c|c|c|}
\hline \multirow[b]{2}{*}{ Index } & \multicolumn{2}{|c|}{ Overall } & \multicolumn{2}{|c|}{ No Apo E4 Alleles } & \multicolumn{2}{|c|}{ 1-2 Apo E4 Alleles } \\
\hline & Case Subjects & Control Subjects & Case Subjects & Control Subjects & Case Subjects & Control Subjects \\
\hline SF-36 PCS & 34 & 50 & 35 & 50 & 33 & 51 \\
\hline SF-36 MCS & 40 & 52 & 41 & 52 & 38 & 53 \\
\hline PSS & 20 & 15 & 20 & 15 & 21 & 15 \\
\hline VAS & 61 & 20 & 60 & 21 & 62 & 19 \\
\hline
\end{tabular}

Apo = apolipoprotein; FMS = fibromyalgia syndrome; MCS = Mental Component Summary score of the SF-36 (higher scores are better); PMRP = Personalized Medicine Research Project; PSS = Perceived Stress Scale score (higher scores suggest greater stress); PCS = Physical Component Summary score of the SF-36 (higher scores are better); SF-36 = Short-Form 36; VAS = visual analogue scale score (higher scores indicate greater average monthly pain).

\section{DISCUSSION}

PCS is a relatively common sequela of mild traumatic brain injury, and research suggests that the Apo E4 genotype is associated not only with an increased risk of developing PCS but with a poorer outcome as well. Given the clinical similarities between FMS and PCS, we wondered whether Apo E4 might also be a genetic risk factor for FMS. In an attempt to answer that question, we conducted this investigation by using the Marshfield Clinic's PMRP biobank. Using strict phenotypic criteria to define FMS, we identified a cohort of 151 subjects diagnosed with FMS (case subjects) and another 300 age- and gendermatched control subjects. Our results reveal a uniform distribution of the various Apo E alleles within the cohort, suggesting that the Apo E4 allele is not an independent genetic risk factor for FMS. However, when one considers the effect of a documented history of previous MVA, crosstab analysis reveals a noticeable increase in the OR when Apo E4 allele status is considered. These results suggest that an interaction occurring between aspects of an individual's history of selected environmental exposures (eg, antecedent MVA) and Apo E4 allele status has the potential to influence their risk of being diagnosed with posttraumatic FMS. Interestingly, although our data suggest that the Apo E4 genotype may enhance susceptibility to the development of posttraumatic FMS, the data do not indicate that Apo E4 influences the severity of the pain, the degree of stress, or the quality of life experienced by individuals with FMS. No statistical association existed between monthly pain severity (as estimated by the VAS) and the physical or mental component summary scores of the SF-36 or the PSS and Apo E4 status.

Although carrying at least one copy of the Apo E4 allele may therefore be a conditional risk factor for FMS, a person's Apo E4 genotype does not appear to influence the severity of symptoms experienced (as reflected by the outcome measures we chose to use in this investigation). As discussed, previously published research has identified Apo E4 as a risk factor for PCS. Given the clinical similarities between FMS and PCS, at the outset of this inquiry we hypothesized that the 2 conditions may share common genetic risk factors. Indeed, our data suggest that Apo E4 is a genetic risk factor for both PCS and FMS. However, there appears to be a significant difference in the physiologic role played by Apo E4 in the 2 conditions. Our observation that Apo E4 does not seem to influence the severity of posttraumatic FMS suggests that the diagnosis is distinct from PCS, at least in regard to the genetic determinants of clinical outcome in general and the role of Apo E4 in particular.

Eventually, genetic and proteomic research will permit us to more fully understand and appreciate the similarities and differences in the pathophysiology underlying FMS and PCS, to say nothing of other potentially related conditions such as chronic fatigue syndrome or overtraining syndrome. Although we suspect that the day will come when clinicians can obtain a battery of laboratory tests to confirm the clinical diagnosis of FMS, at this early stage we cannot recommend that Apo E4 genotyping be incorporated into the workup of an individual with diffuse myofascial pain of uncertain etiology.

The fibromyalgia cases identified in the cohort described in this article were identified retrospectively from the Marshfield Clinic EMR. Because of the variability in documentation we encountered, we could not use the American College of Rheumatology criteria to verify the diagnosis of FMS. In particular, had we insisted on written documentation of myofascial pain at a minimum of 11 tender points above and below the waist and on both sides of the body, we would not have enrolled a sufficient number of cases to permit any analysis of significance. Ultimately, however, we remain confident that our cases do represent FMS because they had each been given the International Classification of Diseases, 9th revision diagnosis of FMS twice within 6 months, they were documented as having widespread myofascial pain, and the collective frequency of associated symptoms was generally consistent with that documented in the literature.

Having demonstrated the utility of the PMRP biobank and the associated EMR in conducting this type of analysis, we propose that additional research be conducted in an effort to identify additional risk factors for and interesting genotypic associations with FMS. In fact, the entire PMRP biobank was recently "fingerprinted" with a panel of 30 medically relevant SNPs [14]. Four SNPs were shown to have a significant statistical association with the FMS phenotype (Table 4). The matrix metallopeptidase 1 allele (MMP1) demonstrated the strongest association $\left(\chi^{2}=8.819, P=.012\right)$. The MMP1 allele previously has been associated with endometriosis and rheumatoid arthritis. Comparison of the frequency distribution of MMPl and the Apo E4 genotypes within the FMS cohort revealed that FMS case 
Table 4. Additional SNPS associated with FMS

\begin{tabular}{|c|c|c|c|}
\hline Allele & SNP & $\begin{array}{l}\text { Observed } \\
\text { Association }\end{array}$ & $\chi^{2}$ Value \\
\hline MMP1 delG & rs1799750 & Case subjects $58 \%-/ C$ & 8.82 \\
\hline HTR2A CC & rs6313 & $\begin{array}{l}\text { Control subjects } 56 \% \\
\text { CT or } \Pi\end{array}$ & 7.41 \\
\hline ELAC2 & rs4792311 & $\begin{array}{l}\text { Case subjects } 2 \times \\
\text { more likely AA }\end{array}$ & 7.39 \\
\hline CBS AA & rs234706 & $\begin{array}{l}\text { Case subjects } 2 \times \\
\text { more likely AA }\end{array}$ & 8.49 \\
\hline
\end{tabular}

$\mathrm{A}=$ adenine; $\mathrm{C}=$ cytidine; $\mathrm{CBS}=$ cystathionine-beta-synthase; del = deleted; ELAC2 = elaC homolog 2; FMS = fibromyalgia syndrome; HTR2A $=5$ hydroxytryptamine receptor $2 \mathrm{~A}$; MMP1 delG = matrix metallopeptidase 1 -deleted G; SNP = single nucleotide polymorphism; T = thymidine.

subjects were most likely to be heterozygous for MMP1 and carry at least one copy of the Apo E4 allele, whereas control subjects were more likely to be homozygous for MMP1 (or $-/-$ ) and have $1-2$ copies of the Apo E4 allele $(P=.002$, likelihood ratio 9.188). Determination of the physiologic significance of an interaction between the Apo E4 and MMP1 alleles (or any of the other SNPs identified in Table 4) must await further analysis, as must determination of whether any of these SNPs can be demonstrated to have an association with PCS or other clinically similar pain syndromes.

\section{CONCLUSION}

Our data suggest that Apo E4 may be a conditional genetic risk factor for FMS in that there appears to be an interaction between individuals with at least one Apo E4 allele and a history of motor vehicular trauma that increases the risk of being diagnosed with FMS. Interestingly, Apo E4 does not seem to play a significant role in the degree to which individuals with posttraumatic fibromyalgia experience pain or perceive stress. Replicating this investigation in a larger population should confirm the significance of the preliminary findings reported here and also permit additional insight into the genetic basis of how conditions that are clinically similar differ from one another.

\section{ACKNOWLEDGMENTS}

Marie Fleisner of the Marshfield Clinic Research Foundation provided assistance in formatting and preparing our final manuscript for submission.

\section{REFERENCES}

1. Arnold LM, Hudson JI, Hess EV, et al. Family study of fibromyalgia. Arthritis Rheum 2004;50:944-952.

2. Kato K, Sullivan PF, Evengård B, Pedersen NL. Chronic widespread pain and its comorbidities: A population-based study. Arch Intern Med 2006;166:1649-1654

3. Johnston KM, McCrory P, Mohtadi NG, Meeuwisse W. Evidence-based review of sport-related concussion: Clinical science. Clin J Sport Med 2001;11:150-159.

4. McCrory P, Johnston KA, Mohtadi NG, Meeuwisse W. Evidence-based review of sport-related concussion: Basic science. Clin J Sport Med 2001;11:160-165.

5. Friedman G, Froom P, Sazbon L. et al. Apolipoprotein E-epsilon4 genotype predicts a poor outcome in survivors of traumatic brain injury. Neurology 1999;52:244-248.

6. Jordan BD, Relkin NR, Ravdin LD, Jacobs AR, Bennett A, Gandy S. Apolipoprotein E epsilon4 associated with chronic traumatic brain injury in boxing. JAMA 1997;278:136-140.

7. Samatovicz RA. Genetics and brain injury: Apolipoprotein E. J Head Trauma Rehabil 2000;15:869-874.

8. Gupta A, Silman AJ. Psychological stress and fibromyalgia: A review of the evidence suggesting a neuroendocrine link. Arthritis Res Ther 2004;6:98-106.

9. Aaron LA, Bradley LA, Alarcon GS, et al. Perceived physical and emotional trauma as precipitating events in fibromyalgia. Associations with health care seeking and disability status but not pain severity. Arthritis Rheum 1997;40:453-460.

10. Martinez-Lavin M. Biology and therapy of fibromyalgia. Stress, the stress response system, and fibromyalgia. Arthritis Res Ther 2007:9:216-222.

11. Buskila D, Sarzi-Puttini P. Biology and therapy of fibromyalgia. Genetic aspects of fibromyalgia syndrome. Arthritis Res Ther 2006;8:218-222.

12. Mortensen $E L, H ø g h ~ P . A$ gender difference in the association between $A P O E$ genotype and age-related cognitive decline. Neurology 2001;57:89-95.

13. McCarty CA, Wilke RA, Giampietro PF, Wesbrook $S$, Caldwell MD. Marshfield Clinic Personalized Medicine Research Project (PMRP): Design, methods and recruitment for a large, population-based biobank. Personalized Med 2005;2:49-79.

14. Cross DS, Ivacic LC, McCarty CA. Development of a fingerprinting panel using medically relevant polymorphisms. BMC Med Genomics 2009;2:17.

15. Cohen S, Kamarck T, Mermelstein R. A global measure of perceived stress. J Health Social Behav 1983;24:385-396.

16. Hoffman DL, Dukes EM. The health status burden of people with fibromyalgia: A review of studies that assessed health status with the SF-36 or the SF-12. Int J Clin Pract 2008;62:115-126.

This CME activity is designated for 1.0 AMA PRA Category 1 Credit ${ }^{\mathrm{TM}}$ and can be completed online at me.aapmr.org. Log on to www.me.aapmr.org, go to Lifelong Learning (CME) and select Journal-based CME from the drop down menu. This activity is FREE to AAPMER members and \$25 for non-members.

\section{CME Question}

Based on the results of this retrospective study, patients with at least one APO E4 allele may have a higher likelihood of developing fibromyalgia syndrome if they have a history of:

a. tobacco use.

b. motor vehicle accident.

c. alcoholism.

d. cumulative trauma disorder.

Answer online at me.aapmr.org 